relative survival of patient groups stratified by Gleason score $(2-6,7,8-10)$ and by PSA levels. Poisson regression analysis revealed associations between PSA and survival: for men with PSA levels $>4 \mathrm{ng} / \mathrm{ml}$, there was a positive correlation between survival and PSA values. However, for men with PSA levels $<4 \mathrm{ng} / \mathrm{ml}$, there was a negative correlation between survival and PSA level, which was most pronounced in men with undifferentiated tumors (Gleason score 8-10).

These results highlight that although most men with low PSA levels have small, indolent tumors, a few may have undifferentiated, aggressive tumors that have lost the ability to secrete PSA.

Original article Sandblom G et al. (2008) The impact of prostate-specific antigen level at diagnosis on the relative survival of 28,531 men with localized carcinoma of the prostate. Cancer 112: 813-819

\section{Successful endoscopic VUR treatment improves children's quality of life}

Endoscopic dextranomer-hyaluronic acid copolymer (DHA) injection is an effective alterative to long-term antibiotic prophylaxis or open surgery for the treatment of vesicouretal reflux (VUR) in children. Nevertheless, the health-related quality of life (HRQOL) benefit of successful endoscopic treatment with DHA is not known; in a new study, Schwentner and colleagues assessed this benefit in a pediatric cohort.

Their retrospective, pilot study included 100 children (mean age 4.46 years; 65 girls) cured of VUR by endoscopic DHA treatment. The Glasgow Children's Benefit Inventory (GCBI) questionnaire-which scores the HRQOL benefits of an intervention on an overall scale from -100 (maximum harm) to +100 (maximum benefit) - was mailed to patients at a mean of $2.16 \pm 1.34$ years after treatment, and 88 parent-completed responses were returned.

Successful treatment had a positive effect on the children's HRQOL (mean total GCBI score $28.4 \pm 20.3$ ); the reported benefits were sustained and independent of critical life events. HRQOL improvement was seen on all four GCBI subscales (emotion, physical health, learning and vitality). Interestingly, sex-specific total $\mathrm{GCBl}$ score and GCBI subscores for emotion, vitality and learning indicated that girls benefited more than boys.

The study found that many areas of the children's lives were improved by successful endoscopic treatment for VUR; however, the authors concede that prospective studies are needed to compare the benefits of endoscopic DHA injection with those of other treatments.

Original article Schwentner C et al. (2008) Health-related quality of life in children with vesicoureteral reflux-impact of successful endoscopic therapy. J Pediatr Urol 4: 20-26

\section{Antioxidants help to reduce short-term damage induced by ESWL}

Extracorporeal shock-wave lithotripsy (ESWL) is the preferred method of treatment for small renal calculi, but is known to induce transient, usually symptomless, free-radical-mediated damage to the kidney and surrounding tissues. Al-Awadi and colleagues postulated that ESWL generates free radicals via a mechanism similar to that of ischemia-reperfusion injury. Accordingly, they investigated whether oral antioxidants (used to mitigate ischemia-reperfusion injury) could alleviate ESWL-induced injury.

The authors enrolled 120 patients with small $(<3 \mathrm{~cm})$ kidney stones, who were randomly assigned to one of three treatment groups: 39 control patients received ESWL only; 40 patients received antioxidants at $2 \mathrm{~h}$ and $8 \mathrm{~h}$ after ESWL; and 41 patients received antioxidants $2 \mathrm{~h}$ before ESWL as well as $2 \mathrm{~h}$ and $8 \mathrm{~h}$ afterwards. Each antioxidant dose consisted of two oral capsules.

At $24 \mathrm{~h}$ after ESWL, antioxidant-treated patients had significantly raised serum ascorbic acid and albumin levels, indicative of improved circulating antioxidant levels, and significantly reduced mean serum concentrations of malondialdehyde, a marker of free-radical-mediated lipid peroxidation (all $P<0.001)$. Antioxidant-treated patients also had a lower a-tocopherol:cholesterol ratio, and lower levels of urinary markers of renal tubular injury (albumin and $\beta 2$ microglobulin), which indicated that ischemia-reperfusion injury was present but less severe in antioxidant-treated patients than in controls. Perhaps surprisingly, antioxidant therapy was still beneficial even in patients who only received it after ESWL. 